\title{
Further results on the cross norm criterion for separability
}

\author{
Oliver Rudolph * \\ Quantum Optics \& Information Group, Istituto Nazionale per la Fisica della Materia \& Dipartimento \\ di Fisica ”A. Volta”, Universita di Pavia, via Bassi 6, I-27100 Pavia, Italy
}

\begin{abstract}
In the present paper the cross norm criterion for separability of density matrices is studied. In the first part of the paper we determine the value of the greatest cross norm for Werner states, for isotropic states and for Bell diagonal states. In the second part we show that the greatest cross norm criterion induces a novel computable separability criterion for bipartite systems. This new criterion is a necessary but in general not a sufficient criterion for separability. It is shown, however, that for all pure states, for Bell diagonal states, for Werner states in dimension $d=2$ and for isotropic states in arbitrary dimensions the new criterion is necessary and sufficient. Moreover, it is shown that for Werner states in higher dimensions $d \geq 3$, the new criterion is only necessary.
\end{abstract}

\section{INTRODUCTION}

The greatest cross norm on the tensor product of the sets of trace class operators on two (or more) Hilbert spaces captures the concept of entanglement in quantum theory in a mathematically natural way: in [1] a separability criterion for mixed quantum states was proven using the greatest cross norm on the tensor product of sets of trace class operators on finite dimensional Hilbert spaces. It was shown that a density operator $\rho$ is separable if and only if the greatest cross norm of $\rho$ is equal to 1. In [2] the value of the greatest cross norm for pure states has been computed in terms of the Schmidt coefficients of the state. In the first part of this paper we determine the value of the greatest cross norm for Werner states and for isotropic states. We use methods to compute entanglement measures under symmetry recently discussed by Vollbrecht and Werner [3] and by Terhal and Vollbrecht [ []. We also clarify the relationship of the greatest cross norm with the robustness of entanglement and determine the value of the greatest cross norm for Bell diagonal states.

In the second part of this paper we introduce and study a novel necessary separability criterion for bipartite systems induced by the greatest cross norm criterion. We show that the new criterion completely characterizes the separability properties of pure states, Bell diagonal states, isotropic states in arbitrary dimensions and Werner states in dimension $d=2$ while in dimension $d \geq 3$ some inseparable Werner states satisfy the criterion as well. Our results imply that the new criterion is neither weaker nor stronger than the Peres-Horodecki positive partial transpose (ppt) criterion [5,6]. [We call a separability criterion (A) weaker than a separability criterion (B) if every state that violates (A) also violates (B).] Our results also show that the new criterion is not weaker than both the reduction criterion for separability [7], and the separability criterion introduced by Nielsen and

*email: rudolph@ fisicavolta.unipv.it 
Kempe [8]. By the results of [9] this also implies that our criterion is not weaker than the entropic separability criteria based on the generalized Rényi and Tsallis entropies. Moreover, violating our criterion does not imply distillability.

This paper is organized as follows: In Section $\amalg$ A we collect some basic definitions and results. In Section ПВ the greatest cross norm is evaluated for operators of rank one. In Section IIC we proceed to compute the value of the greatest cross norm for Werner states, in Section II D for isotropic states and in Section ПIE for Bell diagonal states. In Section ПIF we clarify the relation of the greatest cross norm with the robustness of entanglement introduced in [10]. In Section III] we introduce and study our computable separability criterion.

Throughout this paper the set of trace class operators on some Hilbert space $\mathrm{H}$ is denoted by $\mathrm{T}(\mathrm{H})$, the set of Hilbert-Schmidt operators on $\mathrm{H}$ by $\mathrm{HS}(\mathrm{H})$ and the set of bounded operators on $\mathrm{H}$ by $\mathrm{B}(\mathrm{H})$. A density operator is a positive trace class operator with trace one. We use the Dirac bra/ket notation throughout.

\section{SEPARABILITY AND THE GREATEST CROSS NORM}

\section{A. Preliminaries}

Definition 1 Let $\mathrm{H}_{1}$ and $\mathrm{H}_{2}$ be two Hilbert spaces of arbitrary dimension. A density operator $\rho$ on the tensor product $\mathrm{H}_{1} \otimes \mathrm{H}_{2}$ is called separable if there exist a family $\left\{\omega_{i}\right\}$ of positive real numbers, $a$ family $\left\{\rho_{i}^{(1)}\right\}$ of density operators on $\mathrm{H}_{1}$ and a family $\left\{\rho_{i}^{(2)}\right\}$ of density operators on $\mathrm{H}_{2}$ such that

$$
\rho=\sum_{i} \omega_{i} \rho_{i}^{(1)} \otimes \rho_{i}^{(2)}
$$

where the sum converges in trace class norm. A non-separable state is called entangled.

The Schmidt decomposition is of central importance in the characterization and quantification of entanglement associated with pure states.

Lemma 2 Let $\mathrm{H}_{1}$ and $\mathrm{H}_{2}$ be Hilbert spaces of arbitrary dimension and let $|\psi\rangle \in \mathrm{H}_{1} \otimes \mathrm{H}_{2}$. Then there exist a family of non-negative real numbers $\left\{p_{i}\right\}_{i}$ and orthonormal bases $\left\{\left|a_{i}\right\rangle\right\}_{i}$ and $\left\{\left|b_{i}\right\rangle\right\}_{i}$ of $\mathrm{H}_{1}$ and $\mathrm{H}_{2}$ respectively such that

$$
|\psi\rangle=\sum_{i} \sqrt{p_{i}}\left|a_{i} \otimes b_{i}\right\rangle
$$

The family of positive numbers $\left\{p_{i}\right\}_{i}$ is called the family of Schmidt coefficients of $|\psi\rangle$.

Consider the spaces $\mathrm{T}\left(\mathrm{H}_{1}\right)$ and $\mathrm{T}\left(\mathrm{H}_{2}\right)$ of trace class operators on $\mathrm{H}_{1}$ and $\mathrm{H}_{2}$ respectively. Both spaces are Banach spaces when equipped with the trace class norm $\|\cdot\|_{1}^{(1)}$ or $\|\cdot\|_{1}^{(2)}$ respectively, see, e.g., Schatten [13]. In the sequel we shall drop the superscript and write $\|\cdot\|_{1}$ for both norms, slightly abusing the notation; it will be always clear from the context which norm is meant. The algebraic tensor product $\mathrm{T}\left(\mathrm{H}_{1}\right) \otimes_{\text {alg }} \mathrm{T}\left(\mathrm{H}_{2}\right)$ of $\mathrm{T}\left(\mathrm{H}_{1}\right)$ and $\mathrm{T}\left(\mathrm{H}_{2}\right)$ is defined as the set of all finite linear combinations of elementary tensors $u \otimes v$, i.e., the set of all finite sums $\sum_{i=1}^{n} u_{i} \otimes v_{i}$ where $u_{i} \in \mathrm{T}\left(\mathrm{H}_{1}\right)$ and $v_{i} \in \mathrm{T}\left(\mathrm{H}_{2}\right)$ for all $i$. 
Definition 3 A norm $\|\cdot\|$ on $\mathrm{T}\left(\mathrm{H}_{1}\right) \otimes_{\text {alg }} \mathrm{T}\left(\mathrm{H}_{2}\right)$ is called a subcross norm if $\left\|t_{1} \otimes t_{2}\right\| \leq\left\|t_{1}\right\|_{1}\left\|t_{2}\right\|_{1}$ for all $t_{1} \in \mathrm{T}\left(\mathrm{H}_{1}\right)$ and $t_{2} \in \mathrm{T}\left(\mathrm{H}_{2}\right)$. It is called $a$ cross norm if $\left\|t_{1} \otimes t_{2}\right\|=\left\|t_{1}\right\|_{1}\left\|t_{2}\right\|_{1}$ for all $t_{1} \in \mathrm{T}\left(\mathrm{H}_{1}\right)$ and $t_{2} \in \mathrm{T}\left(\mathrm{H}_{2}\right)$.

It is known that we can define a norm on $\mathrm{T}\left(\mathrm{H}_{1}\right) \otimes_{\mathrm{alg}} \mathrm{T}\left(\mathrm{H}_{2}\right)$ by

$$
\|t\|_{\gamma}:=\inf \left\{\sum_{i=1}^{n}\left\|u_{i}\right\|_{1}\left\|v_{i}\right\|_{1} \mid t=\sum_{i=1}^{n} u_{i} \otimes v_{i}\right\},
$$

where $t \in \mathrm{T}\left(\mathrm{H}_{1}\right) \otimes_{\text {alg }} \mathrm{T}\left(\mathrm{H}_{2}\right)$ and where the infimum runs over all finite decompositions of $t$ into elementary tensors [14].

The norm $\|\cdot\|_{\gamma}$ defined in Equation (2) is born to be subcross and can be shown to be cross (for a proof see, e.g., [14]). Moreover, $\|\cdot\|_{\gamma}$ majorizes any subcross norm on $\mathrm{T}\left(\mathrm{H}_{1}\right) \otimes_{\mathrm{alg}} \mathrm{T}\left(\mathrm{H}_{2}\right)$ and is therefore often also referred to as the greatest cross norm on $\mathrm{T}\left(\mathrm{H}_{1}\right) \otimes_{\mathrm{alg}} \mathrm{T}\left(\mathrm{H}_{2}\right)$. The completion of $\mathrm{T}\left(\mathrm{H}_{1}\right) \otimes_{\text {alg }} \mathrm{T}\left(\mathrm{H}_{2}\right)$ with respect to $\|\cdot\|_{\gamma}$ is denoted by $\mathrm{T}\left(\mathrm{H}_{1}\right) \otimes_{\gamma} \mathrm{T}\left(\mathrm{H}_{2}\right)$. In finite dimensions we have $\mathrm{T}\left(\mathrm{H}_{1}\right) \otimes_{\gamma} \mathrm{T}\left(\mathrm{H}_{2}\right)=\mathrm{T}\left(\mathrm{H}_{1} \otimes \mathrm{H}_{2}\right)[14]$.

In analogy we can also define a cross norm on $\mathrm{HS}\left(\mathrm{H}_{1}\right) \otimes_{\text {alg }} \mathrm{HS}\left(\mathrm{H}_{2}\right)$ by

$$
\|t\|_{g}:=\inf \left\{\sum_{i=1}^{n}\left\|u_{i}\right\|_{2}\left\|v_{i}\right\|_{2} \mid t=\sum_{i=1}^{n} u_{i} \otimes v_{i}\right\}
$$

where $t \in \mathrm{HS}\left(\mathrm{H}_{1}\right) \otimes_{\text {alg }} \mathrm{HS}\left(\mathrm{H}_{2}\right)$ and where the infimum runs over all finite decompositions of $t$ into elementary tensors. $\|\cdot\|_{2}$ denotes the Hilbert-Schmidt norm.

In the following we are mainly interested in the situation where both $\mathrm{H}_{1}$ and $\mathrm{H}_{2}$ are finite dimensional, hence $\mathrm{T}\left(\mathrm{H}_{1}\right)=\mathrm{B}\left(\mathrm{H}_{1}\right)$ and $\mathrm{T}\left(\mathrm{H}_{2}\right)=\mathrm{B}\left(\mathrm{H}_{2}\right)$.

The following theorem demonstrates that $\|\cdot\|_{\gamma}$ captures the concept of entanglement in quantum theory in a mathematically natural way. For a proof see [1].

Theorem 4 Let $\mathrm{H}_{1}$ and $\mathrm{H}_{2}$ be finite dimensional Hilbert spaces and $\rho$ be a density operator on $\mathrm{H}_{1} \otimes \mathrm{H}_{2}$. Then the following statements are equivalent:

- $\rho$ is separable

- $\|\rho\|_{\gamma}=1$.

\section{B. Operators of rank one}

The following proposition is a slight generalization of a proposition that has been proven in [2]. It shows that on pure states $\|\cdot\|_{\gamma}$ can be expressed by the Schmidt coefficients of the state. We reproduce the proof here as the proof method is essential for the results in Section III.

Proposition 5 Let $\mathrm{H}_{1}$ and $\mathrm{H}_{2}$ be finite dimensional Hilbert spaces and let $|\psi\rangle,|\omega\rangle \in \mathrm{H}_{1} \otimes \mathrm{H}_{2}$ be unit vectors and $|\psi\rangle=\sum_{i} \sqrt{p_{i}}\left|\phi_{i}\right\rangle \otimes\left|\chi_{i}\right\rangle$ and $|\omega\rangle=\sum_{j} \sqrt{q_{j}}\left|\alpha_{j}\right\rangle \otimes\left|\beta_{j}\right\rangle$ their Schmidt representations respectively. Here $\left\{\left|\phi_{i}\right\rangle\right\}_{i}$ and $\left\{\left|\alpha_{j}\right\rangle\right\}_{j}$ are orthonormal bases of $\mathrm{H}_{1}$ while $\left\{\left|\chi_{i}\right\rangle\right\}_{i}$ and $\left\{\left|\beta_{j}\right\rangle\right\}_{j}$ are orthonormal bases of $\mathrm{H}_{2}$. Moreover, $p_{i} \geq 0$ and $q_{j} \geq 0$ and $\sum_{i} p_{i}=\sum_{j} q_{j}=1$. Let $S:=|\psi\rangle\langle\omega|$. Then 


$$
\|S\|_{\gamma}=\sum_{i j} \sqrt{p_{i} q_{j}}=\left(\sum_{i} \sqrt{p_{i}}\right)\left(\sum_{i} \sqrt{q_{i}}\right) .
$$

Proof: Without loss of generality we assume that $\mathrm{H}_{1}=\mathrm{H}_{2}$ which can always be achieved by possibly suitably enlarging one of the two Hilbert spaces. Further, we identify $\mathrm{H}_{1}=\mathrm{H}_{2}$ with $\mathbb{C}^{n}$, where $n=\operatorname{dim} \mathrm{H}_{1}$, i.e., we fix an orthonormal basis in $\mathrm{H}_{1}$ which we identify with the canonical real basis in $\mathbb{C}^{n}$. With respect to this canonical real basis in $\mathbb{C}^{n}$ we can define complex conjugates of elements of $\mathrm{H}_{1}$ and the complex conjugate as well as the transpose of a linear operator acting on $\mathrm{H}_{1}$. From the Schmidt decomposition it follows that

$$
S=|\psi\rangle\left\langle\omega\left|=\sum_{i j} \sqrt{p_{i} q_{j}}\right| \phi_{i}\right\rangle\left\langle\alpha_{j}|\otimes| \chi_{i}\right\rangle\left\langle\beta_{j}\right|
$$

From the definition of $\|\cdot\|_{\gamma}$ it is thus obvious that $\|S\|_{\gamma} \leq \sum_{i j} \sqrt{p_{i} q_{j}}$. Now consider the Hilbert space $\mathrm{HS}\left(\mathrm{H}_{1} \otimes \mathrm{H}_{2}\right)$ of Hilbert-Schmidt operators on $\mathrm{H}_{1} \otimes \mathrm{H}_{2}$ equipped with the Hilbert-Schmidt inner product $\langle f \mid g\rangle=\operatorname{tr}\left(f^{\dagger} g\right)$. Equation (屯) induces an operator $\mathfrak{A}_{S}$ on $\mathrm{HS}\left(\mathrm{H}_{1} \otimes \mathrm{H}_{2}\right)$ as follows. Every element $\zeta$ in $\mathrm{HS}\left(\mathrm{H}_{1} \otimes \mathrm{H}_{2}\right)$ can be written $\zeta=\sum_{k} x_{k} \otimes y_{k}$ where $x_{k}$ and $y_{k}$ are trace class operators on $\mathrm{H}_{1}$ and $\mathrm{H}_{2}$ respectively. Then $\mathfrak{A}_{S}$ is defined on $\zeta$ as $\mathfrak{A}_{S}(\zeta):=\sum_{i j k} \sqrt{p_{i} q_{j}}\left\langle\chi_{i}^{*}\left|x_{k}\right| \beta_{j}^{*}\right\rangle\left|\phi_{i}\right\rangle\left\langle\alpha_{j}\right| \otimes y_{k}$ where $\left|\chi_{i}^{*}\right\rangle$ and $\left|\beta_{j}^{*}\right\rangle$ denote, respectively, the complex conjugates of the vectors $\left|\chi_{i}\right\rangle$ and $\left|\beta_{j}\right\rangle$ with respect to the canonical real basis in $\mathbb{C}^{n}$. Proposition 11.1.8 in [15] implies that $\mathfrak{A}_{S}(\zeta)$ is independent of the representation of $\zeta$. Consider a representation $S=\sum_{i=1}^{r} u_{i} \otimes v_{i}$ of $S$ as sum over simple tensors. Denote the transpose of $v_{i}$ by $v_{i}^{T}$. Then the operator defined by

$$
\mathcal{A}_{S}(\zeta):=\sum_{i, k=1}^{r} \operatorname{tr}\left(v_{i}^{T} x_{k}\right) u_{i} \otimes y_{k}
$$

is equal to $\mathfrak{A}_{S}$ (by virtue of Proposition 11.1.8 in [15]). We denote the trace class norm on $\mathrm{T}\left(\mathrm{HS}\left(\mathrm{H}_{1} \otimes \mathrm{H}_{2}\right)\right)$ by $\tau(\cdot)$. The operator $\mathfrak{A}_{S}$ is of trace class and the right hand side of Equation (4) is the so-called polar representation of $\mathfrak{A}_{S}$ which implies $\tau\left(\mathfrak{A}_{S}\right)=\sum_{i j} \sqrt{p_{i} q_{j}}$, see [13]. $\mathfrak{A}_{S}$ admits also many other representations $\mathfrak{A}_{S} \simeq \sum_{i} f_{i} \otimes g_{i}$ with families of operators $\left\{f_{i}\right\}$ and $\left\{g_{i}\right\}$ acting on $\mathrm{H}_{1}$ and $\mathrm{H}_{2}$ respectively. It is known that

$$
\tau\left(\mathfrak{A}_{S}\right)=\inf \left\{\sum_{i}\left\|f_{i}\right\|_{2}\left\|g_{i}\right\|_{2} \mid \mathfrak{A}_{S} \simeq \sum_{i} f_{i} \otimes g_{i}\right\} \leq\|S\|_{\gamma}
$$

where the latter inequality follows from $\|z\|_{2} \leq\|z\|_{1}$ and from the fact that by construction each decomposition of $\mathfrak{A}_{S}$ corresponds in an obvious one-to-one fashion to a decomposition of $S$. For a proof of the first identity in Equation (6) see [13], page 42. This proves the proposition.

Corollary 6 Let $\mathrm{H}_{1}$ and $\mathrm{H}_{2}$ be finite dimensional Hilbert spaces and let $\rho$ be a density operator on $\mathrm{H}_{1} \otimes \mathrm{H}_{2}$. Let $\left\{\left|\phi_{i}\right\rangle\right\}_{i}$ and $\left\{\left|\alpha_{j}\right\rangle\right\}_{j}$ be orthonormal bases of $\mathrm{H}_{1}$ and let $\left\{\left|\chi_{i}\right\rangle\right\}_{i}$ and $\left\{\left|\beta_{j}\right\rangle\right\}_{j}$ be orthonormal bases of $\mathrm{H}_{2}$. If $\rho=\sum_{i j} a_{i j}\left|\phi_{i}\right\rangle\left\langle\alpha_{j}|\otimes| \chi_{i}\right\rangle\left\langle\beta_{j}\right|$, then $\|\rho\|_{\gamma}=\sum_{i j}\left|a_{i j}\right|$. 
Now consider the following expression

$$
\alpha: \mathrm{T}\left(\mathrm{H}_{1} \otimes \mathrm{H}_{2}\right) \rightarrow \mathbb{R}, \alpha(\sigma):=\inf \left\{\sum_{i} \lambda_{i}\left\|S_{i}\right\|_{\gamma} \mid \sigma=\sum_{i} \lambda_{i} S_{i}, \text { where } \lambda_{i} \geq 0, S_{i} \text { of rank } 1\right\}
$$

where the infimum is over all decompositions of $\sigma$ into operators of rank 1. Obviously, $\|\sigma\|_{\gamma} \leq \alpha(\sigma)$ for all $\sigma \in \mathrm{T}\left(\mathrm{H}_{1}\right) \otimes_{\text {alg }} \mathrm{T}\left(\mathrm{H}_{2}\right)$. We first show a little lemma

Lemma 7 Let $\sigma \in \mathrm{T}\left(\mathrm{H}_{1}\right) \otimes_{\text {alg }} \mathrm{T}\left(\mathrm{H}_{2}\right)$, then $\alpha(\sigma)=\|\sigma\|_{\gamma}$

Proof: $\alpha$ is obviously a norm on $\mathrm{T}\left(\mathrm{H}_{1}\right) \otimes_{\text {alg }} \mathrm{T}\left(\mathrm{H}_{2}\right)$. Let $\sigma=\sigma_{1} \otimes \sigma_{2}$ with $\sigma_{1} \in \mathrm{T}\left(\mathrm{H}_{1}\right)$ and $\sigma_{2} \in \mathrm{T}\left(\mathrm{H}_{2}\right)$. Then let $\sigma_{1}=\sum_{i} \lambda_{i}^{(1)} S_{i}^{(1)}$ and $\sigma_{2}=\sum_{j} \lambda_{j}^{(2)} S_{j}^{(2)}$ be the polar decompositions of $\sigma_{1}$ and $\sigma_{2}$ respectively [13]. Then $S_{i}^{(1)}$ and $S_{j}^{(2)}$ are operators of rank 1 for all $i, j$. Thus $\alpha\left(\sigma_{1} \otimes \sigma_{2}\right) \leq \sum_{i j}\left|\lambda_{i}^{(1)} \lambda_{j}^{(2)}\right|=$ $\left\|\sigma_{1} \otimes \sigma_{2}\right\|_{1}$. This proves that $\alpha$ is a subcross norm. As $\|\cdot\|_{\gamma}$ majorizes each subcross seminorm we find that $\alpha(\sigma) \leq\|\sigma\|_{\gamma}$ for all $\sigma \in \mathrm{T}\left(\mathrm{H}_{1} \otimes \mathrm{H}_{2}\right)$. Hence $\alpha(\sigma)=\|\sigma\|_{\gamma}$.

\section{Werner states}

Let $\mathrm{H}$ be a finite dimensional Hilbert space and let $d:=\operatorname{dim} \mathrm{H}>1$. Define

$$
\mathbb{F}:=\sum_{i, j}|i \otimes j\rangle\langle j \otimes i|
$$

where $(|i\rangle)$ is a orthonormal basis of $\mathrm{H}$. Werner states (first considered in [16]) are mixed quantum states in $\mathrm{T}(\mathrm{H} \otimes \mathrm{H})$. They can be parametrized by a real parameter $f$ with $-1 \leq f \leq 1$ and are given by

$$
\rho_{f}:=\frac{1}{d^{3}-d}((d-f) 1+(d f-1) \mathbb{F}) .
$$

Note that $\operatorname{tr}\left(\rho_{f} \mathbb{F}\right)=f$. Let $\mathrm{G}$ be the group of all unitary operators on $\mathrm{H} \otimes \mathrm{H}$ of the form $U \otimes U$ where $U$ is a unitary on $\mathrm{H}$. Then a mixed quantum state is invariant under the action of G, i.e., $\rho=V \rho V^{\dagger}$ for all $V \in \mathrm{G}$ if and only if $\rho=\rho_{f}$ for some $f$, see [16]. Define the twirling operator $\mathbf{P}_{G}$ by

$$
\mathbf{P}_{G}(\sigma) \equiv \int d U(U \otimes U) \sigma\left(U^{\dagger} \otimes U^{\dagger}\right)
$$

where the integration is with respect to the Haar measure of the unitary group on $\mathrm{H}$.

From the definitions of $\mathbf{P}_{G}$ and $\|\cdot\|_{\gamma}$ it readily follows that $\left\|\mathbf{P}_{G}(\sigma)\right\|_{\gamma} \leq\|\sigma\|_{\gamma}$ for all $\sigma \in \mathrm{T}(\mathrm{H} \otimes \mathrm{H})$.

Let $M$ denote the set of operators of rank 1 on $\mathrm{H} \otimes \mathrm{H}$, and consider the expression

$$
\beta\left(\rho_{f}\right):=\inf \left\{\sum_{i} \lambda_{i}\left\|S_{i}\right\|_{\gamma} \mid S_{i} \in M, \lambda_{i} \geq 0, \rho_{f}=\sum_{i} \lambda_{i} \mathbf{P}_{G}\left(S_{i}\right)\right\} .
$$


Lemma 8 Let $f \in[-1,1]$ and let $\rho_{f}$ be the Werner state to $f$, then $\beta\left(\rho_{f}\right)=\left\|\rho_{f}\right\| \gamma$.

Proof: Every admissible decomposition of $\rho_{f}=\sum_{i} \lambda_{i} S_{i}$ in Equation (7) induces via $\rho_{f}=\mathbf{P}_{G}\left(\rho_{f}\right)=$ $\sum_{i} \lambda_{i} \mathbf{P}_{G}\left(S_{i}\right)$ an admissible decomposition in Equation (9). Hence $\beta\left(\rho_{f}\right) \leq \alpha\left(\rho_{f}\right)$. In turn for every decomposition $\rho_{f}=\sum_{i} \lambda_{i} \mathbf{P}_{G}\left(S_{i}\right)$ in Equation (9) we find $\left\|\rho_{f}\right\|_{\gamma} \leq \sum_{i} \lambda_{i}\left\|\mathbf{P}_{G}\left(S_{i}\right)\right\|_{\gamma} \leq \sum_{i} \lambda_{i}\left\|S_{i}\right\|_{\gamma}$. Thus it follows that also $\left\|\rho_{f}\right\|_{\gamma} \leq \beta\left(\rho_{f}\right)$.

We are now ready to compute the greatest cross norm for Werner states.

Theorem 9 Let $\rho_{f}$ be a Werner state, then

$$
\left\|\rho_{f}\right\|_{\gamma}=\left\{\begin{array}{rl}
1 & : \quad \text { for } 0 \leq f \leq 1 \\
1-f & : \quad \text { for }-1 \leq f<0
\end{array} .\right.
$$

Proof: Let $\rho_{f}=\sum_{i} \lambda_{i} \mathbf{P}_{G}\left(S_{i}\right)$ be an admissible decomposition in Equation (9), then we write $S_{i}=$ $\left|\varphi_{i}\right\rangle\left\langle\psi_{i}\right|$ for all $i$. We write the Schmidt decompositions of $\left|\varphi_{i}\right\rangle$ and $\left|\psi_{i}\right\rangle$ as, respectively,

$$
\begin{aligned}
& \left|\varphi_{i}\right\rangle=\sum_{j} \sqrt{p_{j}^{(i)}}\left|a_{j}^{(i)} \otimes b_{j}^{(i)}\right\rangle \\
& \left|\psi_{i}\right\rangle=\sum_{k} \sqrt{q_{k}^{(i)}}\left|d_{k}^{(i)} \otimes e_{k}^{(i)}\right\rangle
\end{aligned}
$$

where $\left(a_{j}^{(i)}\right)_{j},\left(b_{j}^{(i)}\right)_{j},\left(d_{k}^{(i)}\right)_{k}$ and $\left(e_{k}^{(i)}\right)_{k}$ are orthonormal bases of $\mathrm{H}$ respectively for all $i$ and $\sum_{j} p_{j}^{(i)}=\sum_{k} q_{k}^{(i)}=1$ for all $i$. The condition $\operatorname{tr}\left(\rho_{f} \mathbb{F}\right)=f$ reads

$$
f=\sum_{i j k} \lambda_{i} \sqrt{p_{j}^{(i)} q_{k}^{(i)}}\left\langle e_{k}^{(i)} \mid a_{j}^{(i)}\right\rangle\left\langle d_{k}^{(i)} \mid b_{j}^{(i)}\right\rangle .
$$

Thus

$$
\beta\left(\rho_{f}\right)=\inf \left\{\sum_{i j k} \lambda_{i} \sqrt{p_{j}^{(i)} q_{k}^{(i)}} \mid \rho_{f}=\sum_{i} \lambda_{i} \mathbf{P}_{G}\left(\left|\varphi_{i}\right\rangle\left\langle\psi_{i}\right|\right)\right\}
$$

where the infimum is over all decompositions of $\rho_{f}$ of the form $\rho_{f}=\sum_{i} \lambda_{i} \mathbf{P}_{G}\left(\left|\varphi_{i}\right\rangle\left\langle\psi_{i}\right|\right)$ and where $\left(p_{j}^{(i)}\right)_{j}$ and $\left(q_{k}^{(i)}\right)_{k}$ are the Schmidt coefficients of $\left|\varphi_{i}\right\rangle$ and $\left|\psi_{i}\right\rangle$ respectively. Clearly $\beta\left(\rho_{f}\right) \geq$ 1. Now for $0 \leq f \leq 1$ choose $\lambda_{i}=\delta_{i 1}$ and $p_{1}^{(1)}=q_{1}^{(1)}=1$ and $p_{j}^{(1)}=q_{k}^{(1)}=0$ for $j>1$ and $k>1$. Moreover choose $\left\langle e_{1}^{(1)} \mid a_{1}^{(1)}\right\rangle=\left\langle d_{1}^{(1)} \mid b_{1}^{(1)}\right\rangle=\sqrt{f}$, then Equation (11) is satisfied and $\sum_{i j k}\left|\lambda_{i}\right| \sqrt{p_{j}^{(i)} q_{k}^{(i)}}=1$ showing that the infimum is attained $\left\|\rho_{f}\right\|_{\gamma}=\beta\left(\rho_{f}\right)=1$. In the case $-1 \leq$ $f<0$ we note that 


$$
\begin{aligned}
1-f & =\sum_{i j k} \lambda_{i} \sqrt{p_{j}^{(i)} q_{k}^{(i)}}\left(\left\langle d_{k}^{(i)} \mid a_{j}^{(i)}\right\rangle\left\langle e_{k}^{(i)} \mid b_{j}^{(i)}\right\rangle-\left\langle e_{k}^{(i)} \mid a_{j}^{(i)}\right\rangle\left\langle d_{k}^{(i)} \mid b_{j}^{(i)}\right\rangle\right) \\
& \leq \sum_{i j k} \lambda_{i} \sqrt{p_{j}^{(i)} q_{k}^{(i)}}\left|\left\langle d_{k}^{(i)} \mid a_{j}^{(i)}\right\rangle\left\langle e_{k}^{(i)} \mid b_{j}^{(i)}\right\rangle-\left\langle e_{k}^{(i)} \mid a_{j}^{(i)}\right\rangle\left\langle d_{k}^{(i)} \mid b_{j}^{(i)}\right\rangle\right| \\
& \leq \sum_{i j k} \lambda_{i} \sqrt{p_{j}^{(i)} q_{k}^{(i)}} .
\end{aligned}
$$

The last inequality follows readily by considering the Schmidt decomposition of an unnormalized vector of the form $|d \otimes e-e \otimes d\rangle$. Thus in general $\beta\left(\rho_{f}\right) \geq 1-f$. Now choose $\lambda_{i}=\delta_{i 1}$ again and

$$
\left|\varphi_{1}\right\rangle=\left|\psi_{1}\right\rangle \equiv \sqrt{p_{1}^{(1)}}\left|a_{1}^{(1)} \otimes b_{1}^{(1)}\right\rangle-\sqrt{p_{2}^{(1)}}\left|b_{1}^{(1)} \otimes a_{1}^{(1)}\right\rangle
$$

where $p_{1}^{(1)}=1-p_{2}^{(1)}=\frac{1}{2}-\frac{1}{2} \sqrt{1-f^{2}}$ and where $\left|a_{1}^{(1)}\right\rangle$ and $\left|b_{1}^{(1)}\right\rangle$ satisfy $\left\langle b_{1}^{(1)} \mid a_{1}^{(1)}\right\rangle=0$. For this choice Equation (11) is satisfied and we have $1=\sum_{i j} \lambda_{i} \sqrt{p_{j}^{(i)} p_{j}^{(i)}}$ and $-f=\sum_{\substack{i j k \\ j \neq k}} \lambda_{i} \sqrt{p_{j}^{(i)} p_{k}^{(i)}}$. Thus $\left\|\rho_{f}\right\|_{\gamma}=\beta\left(\rho_{f}\right)=1-f$.

\section{Isotropic states}

Again let $\mathrm{H}$ be a finite dimensional Hilbert space with dimension $d:=\operatorname{dim} \mathrm{H}>1$. Consider the group $\widehat{G}$ of a local unitary transformations on $\mathrm{H} \otimes \mathrm{H}$ of the form $U \otimes \bar{U}$ where $U$ is a unitary on $\mathrm{H}$ and $\bar{U}$ denotes the complex conjugate of $U$ with respect to an arbitrary but fixed orthonormal basis in $\mathrm{H}$. The set of states invariant under all elements of $\widehat{G}$ are the so-called isotropic states, see, e.g., [7, 3, 4 ]. The isotropic states can be parametrized by a positive real parameter $F \in[0,1]$ and are given by

$$
\rho_{F} \equiv \frac{1-F}{d^{2}-1}\left(1-\left|\Psi^{+}\right\rangle\left\langle\Psi^{+}\right|\right)+F\left|\Psi^{+}\right\rangle\left\langle\Psi^{+}\right|
$$

Here $\left|\Psi^{+}\right\rangle \equiv \frac{1}{\sqrt{d}} \sum_{i=1}^{d}|i \otimes i\rangle$ and $(|i\rangle)_{i}$ is an arbitrary orthonormal basis in H. We define

$$
\widehat{\mathbb{F}}:=d\left|\Psi^{+}\right\rangle\left\langle\Psi^{+}\left|=\sum_{i j}\right| i \otimes i\right\rangle\langle j \otimes j|
$$

Then $\operatorname{tr}\left(\rho_{F} \widehat{\mathbb{F}}\right)=d F$. We proceed in analogy to Section $\llbracket \mathrm{IC}$ and define the twirling operator $\widehat{\mathbf{P}}_{\widehat{G}}$ for $\widehat{G}$ by

$$
\widehat{\mathbf{P}}_{\widehat{G}}(\sigma):=\int d U(U \otimes \bar{U}) \boldsymbol{\sigma}\left(U^{\dagger} \otimes \bar{U}^{\dagger}\right)
$$

where the integration is again with respect to the Haar measure of the unitary group on H. Let $M$ denote the set of operators of rank 1 on $\mathrm{H} \otimes \mathrm{H}$, then consider the expression

$$
\widehat{\beta}\left(\rho_{F}\right):=\inf \left\{\sum_{i} \lambda_{i}\left\|S_{i}\right\|_{\gamma} \mid S_{i} \in M, \lambda_{i} \geq 0, \rho_{F}=\sum_{i} \lambda_{i} \widehat{\mathbf{P}}_{\widehat{G}}\left(S_{i}\right)\right\} .
$$


Lemma 10 Let $F \in[0,1]$ and let $\rho_{F}$ be the isotropic state to $F$, then $\widehat{\beta}\left(\rho_{F}\right)=\left\|\rho_{F}\right\| \|_{\gamma}$

Proof: Analogous to the proof of Lemma 8 .

Theorem 11 Let $F \in[0,1]$ and $\rho_{F}$ be the isotropic state to $F$, then

$$
\left\|\rho_{F}\right\|_{\gamma}=\left\{\begin{array}{rl}
1 & : \quad \text { for } 0 \leq F \leq \frac{1}{d} \\
d F & : \quad \text { for } \frac{1}{d}<F \leq 1
\end{array} .\right.
$$

Proof: The proof proceeds in analogy to the proof of Theorem 9. Let $\rho_{F}=\sum_{i} \lambda_{i} \widehat{\mathbf{P}}_{\widehat{G}}\left(S_{i}\right)$ be an admissible decomposition in Equation (13), then we write $S_{i}=\left|\varphi_{i}\right\rangle\left\langle\psi_{i}\right|$ for all $i$. We write the Schmidt decompositions of $\left|\varphi_{i}\right\rangle$ and $\left|\psi_{i}\right\rangle$ as $\left|\varphi_{i}\right\rangle=\sum_{j} \sqrt{p_{j}^{(i)}}\left|a_{j}^{(i)} \otimes b_{j}^{(i)}\right\rangle$ and $\left|\psi_{i}\right\rangle=\sum_{k} \sqrt{q_{k}^{(i)}}\left|d_{k}^{(i)} \otimes e_{k}^{(i)}\right\rangle$ where $\left(a_{j}^{(i)}\right)_{j},\left(b_{j}^{(i)}\right)_{j},\left(d_{k}^{(i)}\right)_{k}$ and $\left(e_{k}^{(i)}\right)_{k}$ are orthonormal bases of $\mathrm{H}$ respectively for all $i$ and $\sum_{j} p_{j}^{(i)}=\sum_{k} q_{k}^{(i)}=1$ for all $i$. The condition $\operatorname{tr}\left(\rho_{F} \widehat{\mathbb{F}}\right)=d F$ reads

$$
d F=\sum_{i j k} \lambda_{i} \sqrt{p_{j}^{(i)} q_{k}^{(i)}}\left\langle a_{j}^{(i)^{*}} \mid b_{j}^{(i)}\right\rangle\left\langle d_{k}^{(i)} \mid e_{k}^{(i)^{*}}\right\rangle
$$

where $\left|a_{j}^{(i)^{*}}\right\rangle$ and $\left|e_{k}^{(i)^{*}}\right\rangle$ denote the complex conjugates of $\left|a_{j}^{(i)}\right\rangle$ and $\left|e_{k}^{(i)}\right\rangle$ respectively. Thus

$$
\widehat{\beta}\left(\rho_{F}\right)=\inf \left\{\sum_{i j k} \lambda_{i} \sqrt{p_{j}^{(i)} q_{k}^{(i)}} \mid \rho_{F}=\sum_{i} \lambda_{i} \widehat{\mathbf{P}}_{\widehat{G}}\left(\left|\varphi_{i}\right\rangle\left\langle\psi_{i}\right|\right)\right\}
$$

where the infimum is over all decompositions of $\rho_{f}$ of the form $\rho_{f}=\sum_{i} \lambda_{i} \mathbf{P}_{\widehat{G}}\left(\left|\varphi_{i}\right\rangle\left\langle\psi_{i}\right|\right)$ and where $\left(p_{j}^{(i)}\right)_{j}$ and $\left(q_{k}^{(i)}\right)_{k}$ are the Schmidt coefficients of $\left|\varphi_{i}\right\rangle$ and $\left|\psi_{i}\right\rangle$ respectively. From (15) it follows immediately that $\beta\left(\rho_{F}\right) \geq d F$.

For $d F \geq 1$, consider a state $|\psi\rangle$ of the form $|\psi\rangle=\sum_{i} \sqrt{\mu_{i}}\left|e_{i} \otimes e_{i}\right\rangle$ where $\left\{e_{i}\right\}_{i}$ is an orthonormal basis of $\mathrm{H}$ and where $\left(\sum_{i} \sqrt{\mu_{i}}\right)^{2}=d F$. It has been shown in [] that $\rho_{F}=\widehat{\mathbf{P}}_{\widehat{G}}(|\psi\rangle\langle\psi|)$. Now Proposition 5 implies that $\left\|\rho_{F}\right\|_{\gamma}=\beta\left(\rho_{F}\right)=d F$.

For $0 \leq d F<1$, consider two states $|a\rangle$ and $|b\rangle$ in $\mathrm{H}$ with $\left\langle a^{*} \mid b\right\rangle=\sqrt{d F}$. Again, it has been shown in [凹] that $\rho_{F}=\widehat{\mathbf{P}}_{\widehat{G}}(|a \otimes b\rangle\langle a \otimes b|)$. As by Theorem田we have $\beta\left(\rho_{F}\right) \geq 1$, this proves $\left\|\rho_{F}\right\|_{\gamma}=$ $\beta\left(\rho_{F}\right)=1$.

\section{E. Bell diagonal states}

Consider $\mathbb{C}^{2}$ and let $\{|1\rangle,|2\rangle\}$ be an orthonormal basis of $\mathbb{C}^{2}$. Then the Bell basis of $\mathbb{C}^{2} \otimes \mathbb{C}^{2}$ is given by

$$
\begin{aligned}
& \left|\Psi_{0}\right\rangle \equiv \frac{1}{\sqrt{2}}|11\rangle+|22\rangle,\left|\Psi_{1}\right\rangle \equiv \frac{i}{\sqrt{2}}|12\rangle+|21\rangle \\
& \left|\Psi_{2}\right\rangle \equiv \frac{1}{\sqrt{2}}|21\rangle-|12\rangle,\left|\Psi_{3}\right\rangle \equiv \frac{i}{\sqrt{2}}|11\rangle-|22\rangle .
\end{aligned}
$$


Bell diagonal states are the density operators on $\mathbb{C}^{2} \otimes \mathbb{C}^{2}$ which are diagonal in the Bell basis

$$
\rho=\sum_{i=0}^{3} \lambda_{i}\left|\Psi_{i}\right\rangle\left\langle\Psi_{i}\right|
$$

Bell diagonal states are known to be separable if and only if $\lambda_{i} \leq \frac{1}{2}$ for all $i$, [17, 18].

Theorem 12 Let $\rho \in \mathrm{T}\left(\mathbb{C}^{2} \otimes \mathbb{C}^{2}\right)$ be a Bell diagonal state, i.e., $\rho=\sum_{i=0}^{3} \lambda_{i}\left|\Psi_{i}\right\rangle\left\langle\Psi_{i}\right|$ with $\lambda_{i} \geq 0$ for all $i$. Then

$$
\|\rho\|_{\gamma}=\left\{\begin{array}{rl}
2 \max _{i} \lambda_{i} & : \quad \text { for } \max _{i} \lambda_{i}>\frac{1}{2} \\
1 & : \quad \text { for } \max _{i} \lambda_{i} \leq \frac{1}{2}
\end{array} .\right.
$$

First part of the proof: First consider the case $\max _{i} \lambda_{i} \leq \frac{1}{2}$. In this case there is an explicit decomposition of $\rho$ as a mixture of eight unentangled pure states (see [18] for details). Thus $\|\rho\|_{\gamma}=1$. Now consider the case that $\max _{i} \lambda_{i}>\frac{1}{2}$. In this case there exists an explicit decomposition of $\rho$ as an equal probability mixture of eight entangled pure states, each of which has $\left\{\frac{1}{2}+\frac{1}{2} \sqrt{2 \max _{i} \lambda_{i}-4\left(\max _{i} \lambda_{i}\right)^{2}}, \frac{1}{2}-\frac{1}{2} \sqrt{2 \max _{i} \lambda_{i}-4\left(\max _{i} \lambda_{i}\right)^{2}}\right\}$ as its Schmidt coefficients (again, see [18] for details). From the subadditivity of $\|\cdot\|_{\gamma}$ and Proposition 5 it follows readily that $\|\rho\|_{\gamma} \leq 2 \max _{i} \lambda_{i}$. We postpone the proof for the remaining inequality $\|\rho\|_{\gamma} \geq 2 \max _{i} \lambda_{i}$ until Section IIIE.

\section{F. Relationship with the robustness of entanglement}

Denote the set of Hermitean trace class operators on a Hilbert space $\mathrm{H} \mathrm{by}^{\mathrm{h}}(\mathrm{H})$. A norm closely related to $\|\cdot\|_{\gamma}$ can be defined on $\mathrm{T}^{\mathrm{h}}\left(\mathrm{H}_{1}\right) \otimes_{\text {alg }} \mathrm{T}^{\mathrm{h}}\left(\mathrm{H}_{2}\right)$ by

$$
\|t\|_{S}:=\inf \left\{\sum_{i=1}^{n}\left\|u_{i}\right\|_{1}\left\|v_{i}\right\|_{1} \mid t=\sum_{i=1}^{n} u_{i} \otimes v_{i}\right\}
$$

where $t \in \mathrm{T}^{\mathrm{h}}\left(\mathrm{H}_{1}\right) \otimes_{\text {alg }} \mathrm{T}^{\mathrm{h}}\left(\mathrm{H}_{2}\right)$ and where the infimum runs over all finite decompositions of $t$ into elementary Hermitean tensors. From the definitions of $\|\cdot\|_{\gamma}$ and $\|\cdot\|_{S}$ it is obvious that in general $\|t\|_{\gamma} \leq\|t\|_{S}$ for all Hermitean trace class operators $t$. For a density operator $\sigma$ it is also obvious that $\|\sigma\|_{S}=1$ if and only if $\sigma$ is separable. Clearly, $\|\cdot\|_{S}$ is the greatest cross norm on $\mathrm{T}^{\mathrm{h}}\left(\mathrm{H}_{1}\right) \otimes_{\mathrm{alg}} \mathrm{T}^{\mathrm{h}}\left(\mathrm{H}_{2}\right)$.

Lemma 13 Let $\mathrm{H}$ be a finite dimensional Hilbert space and let $\sigma$ be a Hermitean operator on $\mathrm{H} \otimes \mathrm{H}$, then

$$
\|\sigma\|_{S}=\kappa(\sigma):=\inf \left\{a_{+}+a_{-} \mid \sigma=a_{+} \rho_{+}-a_{-} \rho_{-}, a_{ \pm} \geq 0, \rho_{ \pm} \text {separable density operators }\right\}
$$


Proof: Obviously, for every $\sigma$ there are $a_{ \pm} \geq 0$ and separable density operators $\rho_{ \pm}$such that $\sigma=a_{+} \rho_{+}-a_{-} \rho_{-}$. [It is always possible to write $\sigma$ as a sum of Hermitean simple tensors $\sigma=\sum_{i} x_{i} \otimes y_{i}$; to get the desired decomposition just decompose all $x_{i}$ and $y_{i}$ into their positive and negative parts and rearrange terms]. The inequality $\|\sigma\|_{S} \leq \kappa(\sigma)$ is obvious. If $\sigma=\sigma_{1} \otimes \sigma_{2}$, then $\kappa(\sigma) \leq\left\|\sigma_{1}\right\|_{1}\left\|\sigma_{2}\right\|_{1}$. Thus $\kappa$ is a subcross norm and thus $\kappa(\sigma) \leq\|\sigma\|_{S}$ for all Hermitean $\sigma$.

For a density operator $\sigma$ the quantity $E_{R}(\sigma) \equiv \frac{1}{2}\left(\|\sigma\|_{S}-1\right)$ is called robustness of entanglement [10], see also [19]. The robustness of entanglement has the physical meaning of the minimal amount of separable noise that destroys the entanglement of a given state.

Proposition 14 Let $\mathrm{H}$ be a finite dimensional Hilbert space. Then the robustness of entanglement and the greatest cross norm on $\mathrm{T}(\mathrm{H} \otimes \mathrm{H})$ are related by

$$
E_{R}(\sigma) \geq\|\sigma\|_{\gamma}-1
$$

where $\sigma$ is a positive trace class operator with trace one.

Proof: The analogue of Lemma 7 holds for $\|\cdot\|_{S}$. Let $|\psi\rangle \in \mathrm{H} \otimes \mathrm{H}$ and let $P_{\psi}=|\psi\rangle\langle\psi|$. In [10] it has been shown that $\left\|P_{\psi}\right\|_{S}=2\left(\sum_{i} \sqrt{p_{i}}\right)^{2}-1$. Thus Proposition 5 implies that $\left\|P_{\psi}\right\|_{S}=2\left\|P_{\psi}\right\|_{\gamma}-1$. Therefore Lemma 7 and the analogue statement for $\|\cdot\|_{S}$ imply that $\|\sigma\|_{S} \geq 2\|\sigma\|_{\gamma}-1$ for all positive Hermitean $\sigma$ with trace one. This proves the proposition.

For projection operators we have equality in Equation ([18). Moreover, in [19] Vidal and Werner computed the robustness of entanglement for density operators with symmetry. The results of Vidal and Werner show that for Werner and isotropic states there is also an equality in Equation (18). However, a proof of whether or not equality holds in (18) in general has not been found by this author.

\section{A COMPUTABLE SEPARABILITY CRITERION}

\section{A. Formulation of the criterion}

Every finite dimensional Hilbert space $\mathrm{H}$ is isomorphic to $\mathbb{C}^{n}$, with $n=\operatorname{dim}(\mathrm{H})$. This corresponds to identifying a fixed orthonormal basis in $\mathrm{H}$ with the canonical real basis in $\mathbb{C}^{n}$. In $\mathbb{C}^{n}$ there is a notion of complex conjugation. We denote the complex conjugate of $|\psi\rangle \in \mathbb{C}^{n}$ by $\left|\psi^{*}\right\rangle$.

Proposition 15 Let $\mathrm{K}_{1} \simeq \mathbb{C}^{n}$ and $\mathrm{K}_{2} \simeq \mathbb{C}^{m}$ be finite dimensional Hilbert spaces. There is a oneto-one correspondence between states $|\psi\rangle \in \mathrm{K}_{1} \otimes \mathrm{K}_{2}$ and Hilbert-Schmidt operators $A: \mathrm{K}_{2} \rightarrow \mathrm{K}_{1}$ according to the rule: let $|\psi\rangle=\sum_{i j} c_{i j}\left|a_{i}\right\rangle \otimes\left|b_{j}\right\rangle$ be a decomposition of $|\psi\rangle$ in terms of orthonormal bases $\left\{\left|a_{i}\right\rangle\right\}$ and $\left\{\left|b_{j}\right\rangle\right\}$ of $\mathrm{K}_{1}$ and $\mathrm{K}_{2}$ respectively. Then $A(\psi)$ is given by $A(\psi)=\sum_{i j} c_{i j}\left|a_{i}\right\rangle\left\langle b_{j}^{*}\right|$. Conversely, if $A=\sum_{i j} c_{i j}\left|a_{i}\right\rangle\left\langle b_{j}\right|$ for some orthonormal bases $\left\{\left|a_{i}\right\rangle\right\}$ and $\left\{\left|b_{j}\right\rangle\right\}$ of $\mathrm{K}_{1}$ and $\mathrm{K}_{2}$ respectively, then $\left|\psi_{A}\right\rangle=\sum_{i j} c_{i j}\left|a_{i}\right\rangle \otimes\left|b_{j}^{*}\right\rangle$. 
Proof: We only need to show that $A(\psi)$ is well-defined and independent of the decomposition of $|\psi\rangle$ and similarly that $\left|\psi_{A}\right\rangle$ is independent of the representation of $A$ chosen. But this follows immediately from, e.g., Proposition 11.1 .8 in [15].

Corollary 16 Let $\mathrm{K}_{1} \simeq \mathbb{C}^{n}$ and $\mathrm{K}_{2} \simeq \mathbb{C}^{m}$ be finite dimensional Hilbert spaces. The one-to-one correspondence between pure states $|\psi\rangle \in \mathrm{K}_{1} \otimes \mathrm{K}_{2}$ and Hilbert-Schmidt operators $A: \mathrm{K}_{2} \rightarrow \mathrm{K}_{1}$ from Proposition 15 is isometric, i.e., $\left\langle A\left(\psi_{1}\right) \mid A\left(\psi_{2}\right)\right\rangle_{H S}=\left\langle\psi_{1} \mid \psi_{2}\right\rangle$ and $\left\langle\psi_{A} \mid \psi_{B}\right\rangle=\langle A \mid B\rangle_{H S}$.

Proof: Denote the canonical real bases of $\mathrm{K}_{1}$ and $\mathrm{K}_{2}$ by $\left\{\left|e_{i}\right\rangle\right\}_{i}$ and $\left\{\left|f_{j}\right\rangle\right\}_{j}$ respectively. Let $\left|\psi_{1}\right\rangle=$ $\sum_{i j} c_{i j}\left|e_{i}\right\rangle \otimes\left|f_{j}\right\rangle$ and $\left|\psi_{2}\right\rangle=\sum_{p q} d_{p q}\left|e_{p}\right\rangle \otimes\left|f_{q}\right\rangle$ the decompositions of $\left|\psi_{1}\right\rangle \in \mathrm{K}_{1}$ and $\left|\psi_{2}\right\rangle \in \mathrm{K}_{2}$ in terms of these bases. Then $A\left(\psi_{1}\right)=\sum_{i j} c_{i j}\left|e_{i}\right\rangle\left\langle f_{j}^{*}\left|=\sum_{i j} c_{i j}\right| e_{i}\right\rangle\left\langle f_{j}\right|$ and $A\left(\psi_{2}\right)=\sum_{p q} d_{p q}\left|e_{p}\right\rangle\left\langle f_{q}^{*}\right|=$ $\sum_{p q} d_{p q}\left|e_{p}\right\rangle\left\langle f_{q}\right|$. Therefore $\left\langle A\left(\psi_{1}\right) \mid A\left(\psi_{2}\right)\right\rangle_{H S}=\operatorname{tr}\left(A^{\dagger}\left(\psi_{1}\right) A\left(\psi_{2}\right)\right)=\sum_{i j p q} c_{i j}^{*} d_{p q}\left\langle f_{q} \mid f_{j}\right\rangle\left\langle e_{i} \mid e_{p}\right\rangle=$ $\sum_{i j p q} c_{i j}^{*} d_{p q}\left\langle f_{j} \mid f_{q}\right\rangle\left\langle e_{i} \mid e_{p}\right\rangle=\left\langle\psi_{1} \mid \psi_{2}\right\rangle$. This proves the corollary.

To derive the next theorem we use Proposition 15 in the case that $K_{1}$ and $K_{2}$ are the spaces of Hilbert-Schmidt operators on some other Hilbert spaces $\mathrm{H}_{1}$ and $\mathrm{H}_{2}$ respectively, i.e., $\mathrm{K}_{1}=\mathrm{HS}\left(\mathrm{H}_{1}\right)$ and $\mathrm{K}_{2}=\mathrm{HS}\left(\mathrm{H}_{2}\right)$.

Theorem 17 Let $\mathrm{H}_{1}$ and $\mathrm{H}_{2}$ be finite dimensional Hilbert spaces and let $\mathrm{K}_{1}=\mathrm{HS}\left(\mathrm{H}_{1}\right) \simeq \mathbb{C}^{n}$ and $\mathrm{K}_{2}=\mathrm{HS}\left(\mathrm{H}_{2}\right) \simeq \mathbb{C}^{m}$ be the spaces of Hilbert-Schmidt operators on $\mathrm{H}_{1}$ and $\mathrm{H}_{2}$ respectively. Then there exists a one-to-one correspondence between Hilbert-Schmidt operators $T \in \mathrm{HS}\left(\mathrm{H}_{1} \otimes \mathrm{H}_{2}\right)$ and Hilbert-Schmidt operators $\mathfrak{A}(T): \mathrm{HS}\left(\mathrm{H}_{2}\right) \rightarrow \mathrm{HS}\left(\mathrm{H}_{1}\right)$ analogous to the correspondence in Proposition 15 .

Proof: It is well-known that HS(H) furnished with the Hilbert-Schmidt inner product $\langle A \mid B\rangle_{H S} \equiv$ $\operatorname{tr}\left(A^{\dagger} B\right)$ is a Hilbert space. Therefore Theorem 17 is an immediate consequence of Proposition 15.

The correspondence described in Proposition 15 and Theorem 17 has been known and applied in the quantum optics literature for some time, see [20,21] and references therein for more details.

In the sequel we always assume without loss of generality that $\mathrm{H}_{1}=\mathrm{H}_{2}$ and as in the proof of Proposition 5 we denote the trace class norm of $\mathfrak{A}(T)$ by $\tau(\mathfrak{A}(T))$.

Corollary 18 With the notation from Theorem 17 let $T \in \mathrm{HS}(\mathrm{H} \otimes \mathrm{H})$ be a Hilbert-Schmidt operator on $\mathrm{H} \otimes \mathrm{H}$. Then there exist a family $\left\{\lambda_{i}\right\}_{i}$ of non-negative real numbers, orthonormal bases $\left\{E_{i}\right\}_{i}$ and $\left\{F_{i}\right\}_{i}$ of $\mathrm{HS}(\mathrm{H})$ and $\mathrm{HS}(\mathrm{H})$ respectively such that

$$
T=\sum_{i} \lambda_{i} E_{i} \otimes F_{i}
$$

Moreover we have $\tau(\mathfrak{A}(T))=\sum_{i} \lambda_{i}$.

Proof: This is an immediate consequence of Proposition 15 and Theorem 17.

Corollary 18 can be viewed as an analogue of the Schmidt decomposition for density operators. 
We now apply our results to the separability problem for density operators on $\mathrm{H} \otimes \mathrm{H}$. It is known that for any operator $A: \mathrm{HS}(\mathrm{H}) \rightarrow \mathrm{HS}(\mathrm{H})$ the following identity holds (see [13], page 42, and also Equation (6)

$$
\left.\tau(A) \equiv \inf \left\{\sum_{i}\left\|f_{i}\right\|_{2}\left\|g_{i}\right\|_{2}\left|A=\sum_{i}\right| f_{i}\right\rangle\left\langle g_{i}|,| f_{i}\right\rangle,\left|g_{i}\right\rangle \in \mathrm{HS}(\mathrm{H})\right\}
$$

where the infimum is over all finite decompositions of $A$ into simple tensors of Hilbert-Schmidt operators. The next Proposition is our new necessary separability criterion.

Proposition 19 Let $\mathrm{H}$ be a finite dimensional Hilbert space. Let $\rho \in \mathrm{T}(\mathrm{H} \otimes \mathrm{H})$ be a density operator. If $\rho$ is separable, then

$$
\tau(\mathfrak{A}(\rho)) \leq 1
$$

Proof: This follows immediately from Equation (20) and Theorem 4 .

Corollary 20 Let $\mathrm{H}$ be a finite dimensional Hilbert space. Let $|\psi\rangle \in \mathrm{H} \otimes \mathrm{H}$ be a pure state. $|\psi\rangle$ is separable if and only if $\tau\left(\mathfrak{A}\left(P_{\psi}\right)\right)=1$.

Proof: This follows immediately from the proof of Proposition 5 .

Remark 21 To check whether the separability criterion in Proposition 19 is satisfied by a given density operator $\rho$ reduces to the evaluation of the trace class norm of the Hilbert-Schmidt operator $\mathfrak{A}(\rho)$. This is completely straightforward using standard linear algebra packages and accordingly Equation (21) is a computable separability criterion for density operators.

In the next two subsections we compute $\tau(\mathfrak{A}(\rho))$ in the situations that $\rho$ is an isotropic state or a Werner state respectively. Moreover, in the subsequent subsections we study other families of states for which Equation (21) can be computed.

\section{B. Isotropic states}

We continue to use our notation from Section $\llbracket$ II. We rewrite $\rho_{F}$ as

$$
\rho_{F}=\frac{1-\alpha_{F}}{d^{2}} I+\alpha_{F}\left|\Psi^{+}\right\rangle\left\langle\Psi^{+}\right|
$$

where $\alpha_{F} \equiv \frac{d^{2} F-1}{d^{2}-1}$. We prove

Proposition 22 Let $F \in[0, d]$ and $\rho_{F}$ be the corresponding isotropic state, then

$$
\tau\left(\mathfrak{A}\left(\rho_{F}\right)\right)=\left\{\begin{array}{rl}
d F & : \quad \text { for } \frac{1}{d^{2}} \leq F \leq 1 \\
\frac{2}{d}-d F & : \quad \text { for } 0 \leq F<\frac{1}{d^{2}}
\end{array} .\right.
$$


For the proof of this proposition we need Ferrers' formula [22].

Lemma 23 (Ferrers) Let $0<n \in \mathbb{N}$ and $a_{1}, a_{2}, \ldots, a_{n} \in \mathbb{C} \backslash\{0\}$, then

$$
\operatorname{det}\left(\begin{array}{ccccc}
1+a_{1} & 1 & 1 & \cdots & 1 \\
1 & 1+a_{2} & 1 & \cdots & 1 \\
1 & 1 & 1+a_{3} & \cdots & 1 \\
\vdots & \vdots & \vdots & \ddots & \vdots \\
1 & 1 & 1 & \cdots & 1+a_{n}
\end{array}\right)=a_{1} a_{2} \cdots a_{n}\left(1+\frac{1}{a_{1}}+\cdots+\frac{1}{a_{n}}\right)
$$

Ferrers' formula follows readily by induction.

Proof of Proposition 22: Denote by $\{|i\rangle\}_{i}$ the canonical real basis of $\mathbb{C}^{d}$. From Equation (22) it follows that

$$
\mathfrak{A}\left(\rho_{F}\right)=\frac{\alpha_{F}}{d} \sum_{i j}\left|E_{i j}\right\rangle\left\langle E_{i j}\left|+\frac{1-\alpha_{F}}{d^{2}} \sum_{i j}\right| E_{i i}\right\rangle\left\langle E_{j j}\right|
$$

where $E_{i j} \equiv|i\rangle\langle j|$ and where we use the notation $\left|E_{i j}\right\rangle$ for $E_{i j}$ to stress that we think of $E_{i j}$ as an element of $\operatorname{HS}\left(\mathbb{C}^{d}\right)$. Thus $\mathfrak{A}\left(\rho_{F}\right)^{\dagger}=\mathfrak{A}\left(\rho_{F}\right)$ and

$$
\begin{aligned}
\mathfrak{A}\left(\rho_{F}\right)^{\dagger} \mathfrak{A}\left(\rho_{F}\right) & =\frac{\alpha_{F}^{2}}{d^{2}} \sum_{i j}\left|E_{i j}\right\rangle\left\langle E_{i j}\left|+\frac{1-\alpha_{F}^{2}}{d^{3}} \sum_{i j}\right| E_{i i}\right\rangle\left\langle E_{j j}\right| \\
& =\frac{\alpha_{F}^{2}}{d^{2}} \sum_{\substack{i j \\
i \neq j}}\left|E_{i j}\right\rangle\left\langle E_{i j}\left|+\frac{\alpha_{F}^{2}}{d^{2}} \sum_{i}\right| E_{i i}\right\rangle\left\langle E_{i i}\left|+\frac{1-\alpha_{F}^{2}}{d^{3}} \sum_{i j}\right| E_{i i}\right\rangle\left\langle E_{j j}\right| .
\end{aligned}
$$

From the formula (23) we see that $\frac{\alpha_{F}^{2}}{d^{2}}$ is an Eigenvalue of $\mathfrak{A}\left(\rho_{F}\right)^{\dagger} \mathfrak{A}\left(\rho_{F}\right)$ with multiplicity (at least) $d^{2}-d$. The second two terms in Equation (23) act only on the subspace $\mathcal{S}_{d}$ spanned by the elements $\left|E_{i i}\right\rangle$. The matrix representation of the second two terms in Equation (23) in the basis $\left\{\left|E_{i i}\right\rangle\right\}_{i}$ of $\mathcal{S}_{d}$ is

$$
\frac{\alpha_{F}^{2}}{d^{2}} \sum_{i}\left|E_{i i}\right\rangle\left\langle E_{i i}\left|+\frac{1-\alpha_{F}^{2}}{d^{3}} \sum_{i j}\right| E_{i i}\right\rangle\left\langle E_{j j}\right| \simeq \frac{1-\alpha_{F}^{2}}{d^{3}}\left(\begin{array}{cccccc}
1+\frac{d \alpha_{F}^{2}}{1-\alpha_{F}^{2}} & 1 & 1 & \cdots & 1 \\
1 & 1+\frac{d \alpha_{F}^{2}}{1-\alpha_{F}^{2}} & 1 & \cdots & 1 \\
1 & 1 & 1+\frac{d \alpha_{F}^{2}}{1-\alpha_{F}^{2}} & \cdots & 1 \\
\vdots & \vdots & \vdots & \ddots & \vdots \\
1 & 1 & 1 & \cdots & 1+\frac{d \alpha_{F}^{2}}{1-\alpha_{F}^{2}}
\end{array}\right) .
$$

The Eigenvalues of this matrix can readily be evaluated with the help of Lemma 23 and are found to be $\lambda_{1}=\frac{\alpha_{F}^{2}}{d^{2}}$ with $d-1$-fold multiplicity and $\lambda_{2}=\frac{1}{d^{2}}$ (with multiplicity one). Therefore adding the absolute values of the square roots of all Eigenvalues, we arrive at $\tau\left(\mathfrak{A}\left(\rho_{F}\right)\right)=\left|\alpha_{F}\right|\left(d-\frac{1}{d}\right)+\frac{1}{d}$. This proves the Proposition. 
Corollary $24 \operatorname{tr}\left(\mathfrak{A}\left(\rho_{F}\right)\right)=d F$ and $\left\|\rho_{F}\right\|_{g}=\sqrt{\alpha_{F}^{2} \frac{d^{2}-1}{d^{2}}+\frac{1}{d^{2}}}$.

Proposition 22 implies that an isotropic state $\rho_{F}$ is separable if and only if $\tau\left(\mathfrak{A}\left(\rho_{F}\right)\right) \leq 1$.

\section{Werner states}

Proposition 25 Let $f \in[-1,1]$ and $\rho_{f}$ be the corresponding Werner state, then

$$
\tau\left(\mathfrak{A}\left(\rho_{f}\right)\right)=\left\{\begin{array}{rl}
\frac{2}{d}-f & : \quad \text { for }-1 \leq f \leq \frac{1}{d} \\
f & : \quad \text { for } 1 \geq f \geq \frac{1}{d}
\end{array} .\right.
$$

Proof: We write

$$
\mathfrak{A}\left(\rho_{f}\right)=\frac{d-f}{d^{3}-d} \sum_{i j}\left|E_{i i}\right\rangle\left\langle E_{j j}\left|+\frac{d f-1}{d^{3}-d} \sum_{i j}\right| E_{i j}\right\rangle\left\langle E_{j i}\right| .
$$

An argument as above shows that $\mathfrak{A}\left(\rho_{f}\right)^{\dagger} \mathfrak{A}\left(\rho_{f}\right)$ has the simple Eigenvalue $\lambda_{0}=\frac{1}{d^{2}}$ and the degenerate Eigenvalue $\lambda_{1}=\frac{(d f-1)^{2}}{\left(d^{3}-d\right)^{2}}$ with multiplicity $d^{2}-1$. This shows that $\tau\left(\mathfrak{A}\left(\rho_{f}\right)\right)=\frac{|d f-1|}{d}+\frac{1}{d}$.

Proposition 25 shows that the criterion in Equation (21) is satisfied whenever $f \in\left[\frac{2}{d}-1,1\right]$. This proves that for Werner states the criterion in Equation (21) is exact if and only if $d=2$. In higher dimension $d \geq 3$ there will always be inseparable Werner states (i.e., those corresponding to $f \in\left[\frac{2}{d}-1,0[\right.$ ) which satisfy the criterion in Proposition 19 while other inseparable Werner states (i.e., those corresponding to $f \in\left[-1, \frac{2}{d}-1[\right.$ ) violate it.

Corollary $26 \operatorname{tr}\left(\mathfrak{A}\left(\rho_{f}\right)\right)=\frac{f+1}{d+1}$ and $\left\|\mathfrak{A}\left(\rho_{f}\right)\right\|_{g}=\sqrt{\frac{1+f^{2}}{d^{2}-1}-\frac{2 f}{d\left(d^{2}-1\right)}}$.

\section{A two qubit example}

Denote the canonical real basis in $\mathbb{C}^{2}$ by $\{|0\rangle,|1\rangle\}$ and consider the following family of states on $\mathbb{C}^{2} \otimes \mathbb{C}^{2}$

$$
\rho_{p} \equiv p|00\rangle\langle 00|+(1-p)| \Phi\rangle\langle\Phi|,
$$

where $0 \leq p \leq 1$ and $|\Phi\rangle=\frac{1}{\sqrt{2}}(|01\rangle+|10\rangle)$. Then

$$
\mathfrak{A}\left(\rho_{p}\right)=p\left|E_{00}\right\rangle\left\langle E_{00}\right|+\frac{1-p}{2}\left(\left|E_{00}\right\rangle\left\langle E_{11}|+| E_{11}\right\rangle\left\langle E_{00}|+| E_{10}\right\rangle\left\langle E_{01}|+| E_{01}\right\rangle\left\langle E_{10}\right|\right)
$$

It is straightforward to compute the trace class norm of $\mathfrak{A}\left(\rho_{p}\right)$. The result is 


$$
\begin{aligned}
& \tau\left(\mathfrak{A}\left(\rho_{p}\right)\right)=1-p+\sqrt{\frac{p^{2}}{2}+\frac{(1-p)^{2}}{4}+\frac{p}{2} \sqrt{p^{2}+(1-p)^{2}}}+\sqrt{\frac{p^{2}}{2}+\frac{(1-p)^{2}}{4}-\frac{p}{2} \sqrt{p^{2}+(1-p)^{2}}} \\
& \geq 1-p+p \sqrt{1+\frac{(1-p)^{2}}{2 p^{2}}} \geq 1
\end{aligned}
$$

with equality if and only if $p=1$. Therefore Equation (21) implies that $\rho_{p}$ is separable if and only if $p=1$.

\section{E. Bell diagonal states}

We continue to use our notation from Section $\Pi \mathrm{E}$ but assume now that (without loss of generality) $\{|1\rangle,|2\rangle\}$ denotes the canonical real basis in $\mathbb{C}^{2}$. Let $\rho$ be a Bell diagonal state, i.e., a density operator on $\mathbb{C}^{2} \otimes \mathbb{C}^{2}$ of the form

$$
\rho=\sum_{i=0}^{3} \lambda_{i}\left|\Psi_{i}\right\rangle\left\langle\Psi_{i}\right|
$$

Then

$$
\begin{aligned}
\mathfrak{A}(\rho)= & \frac{\lambda_{0}+\lambda_{3}}{2}\left(\left|E_{11}\right\rangle\left\langle E_{11}|+| E_{22}\right\rangle\left\langle E_{22}\right|\right)+\frac{\lambda_{1}+\lambda_{2}}{2}\left(\left|E_{11}\right\rangle\left\langle E_{22}|+| E_{22}\right\rangle\left\langle E_{11}\right|\right) \\
& +\frac{\lambda_{0}-\lambda_{3}}{2}\left(\left|E_{12}\right\rangle\left\langle E_{12}|+| E_{21}\right\rangle\left\langle E_{21}\right|\right)+\frac{\lambda_{1}-\lambda_{2}}{2}\left(\left|E_{12}\right\rangle\left\langle E_{21}|+| E_{21}\right\rangle\left\langle E_{12}\right|\right) .
\end{aligned}
$$

Hence

$$
\begin{aligned}
\mathfrak{A}(\rho)^{\dagger} \mathfrak{A}(\rho)= & \frac{\left(\lambda_{0}+\lambda_{3}\right)^{2}+\left(\lambda_{1}+\lambda_{2}\right)^{2}}{4}\left(\left|E_{11}\right\rangle\left\langle E_{11}|+| E_{22}\right\rangle\left\langle E_{22}\right|\right) \\
& +\frac{\left(\lambda_{0}+\lambda_{3}\right)\left(\lambda_{1}+\lambda_{2}\right)}{2}\left(\left|E_{11}\right\rangle\left\langle E_{22}|+| E_{22}\right\rangle\left\langle E_{11}\right|\right) \\
& +\frac{\left(\lambda_{0}-\lambda_{3}\right)^{2}+\left(\lambda_{1}-\lambda_{2}\right)^{2}}{4}\left(\left|E_{12}\right\rangle\left\langle E_{12}|+| E_{21}\right\rangle\left\langle E_{21}\right|\right) \\
& +\frac{\left(\lambda_{1}-\lambda_{2}\right)\left(\lambda_{0}-\lambda_{3}\right)}{2}\left(\left|E_{12}\right\rangle\left\langle E_{21}|+| E_{21}\right\rangle\left\langle E_{12}\right|\right) .
\end{aligned}
$$

It is straightforward to compute the trace class norm of $\mathfrak{A}(\rho)$. The result is

$$
\begin{aligned}
& \tau(\mathfrak{A}(\rho))=\frac{1}{2}\left(1+\left|\lambda_{0}+\lambda_{3}-\lambda_{1}-\lambda_{2}\right|+\left|\lambda_{1}-\lambda_{2}\right|+\left|\lambda_{0}-\lambda_{3}\right|+|| \lambda_{0}-\lambda_{3}|-| \lambda_{1}-\lambda_{2}||\right)
\end{aligned}
$$

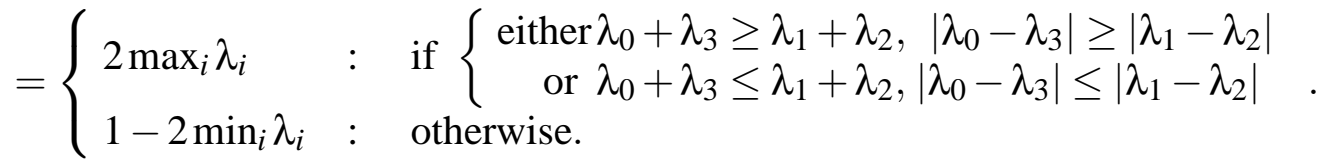

To see Equation (24-b) note that 
- if $\lambda_{0}+\lambda_{3} \geq \lambda_{1}+\lambda_{2}$ and $\left|\lambda_{0}-\lambda_{3}\right| \geq\left|\lambda_{1}-\lambda_{2}\right|$, then $\max _{i} \lambda_{i}=\max \left\{\lambda_{0}, \lambda_{3}\right\}$. Similarly, if $\lambda_{1}+\lambda_{2} \geq \lambda_{0}+\lambda_{3}$ and $\left|\lambda_{1}-\lambda_{2}\right| \geq\left|\lambda_{0}-\lambda_{3}\right|$, then $\max _{i} \lambda_{i}=\max \left\{\lambda_{1}, \lambda_{2}\right\}$.

- Conversely, if $\lambda_{0}+\lambda_{3} \geq \lambda_{1}+\lambda_{2}$ and $\left|\lambda_{0}-\lambda_{3}\right|<\left|\lambda_{1}-\lambda_{2}\right|$, we find $\min _{i} \lambda_{i}=\min \left\{\lambda_{1}, \lambda_{2}\right\}$. Similarly, if $\lambda_{1}+\lambda_{2} \geq \lambda_{0}+\lambda_{3}$ and $\left|\lambda_{1}-\lambda_{2}\right|<\left|\lambda_{0}-\lambda_{3}\right|$, we find $\min _{i} \lambda_{i}=\min \left\{\lambda_{0}, \lambda_{3}\right\}$.

- Note also that if $\max _{i} \lambda_{i} \geq \frac{1}{2}$, then we have either the situation that $\lambda_{0}+\lambda_{3} \geq \lambda_{1}+\lambda_{2}$ and $\left|\lambda_{0}-\lambda_{3}\right| \geq\left|\lambda_{1}-\lambda_{2}\right|$ or that $\lambda_{0}+\lambda_{3} \leq \lambda_{1}+\lambda_{2}$ and $\left|\lambda_{0}-\lambda_{3}\right| \leq\left|\lambda_{1}-\lambda_{2}\right|$.

Proof: To see this, assume without loss of generality that $\lambda_{0}=\max _{i} \lambda_{i}=\frac{1}{2}+\delta$ for some $\delta \geq 0$. Write $\lambda_{3}=\lambda_{0}-\varepsilon$, for $\varepsilon \geq 0$. Now assume that $\lambda_{0}+\lambda_{3} \geq \lambda_{1}+\lambda_{2}$, but $\left|\lambda_{0}-\lambda_{3}\right|<\left|\lambda_{1}-\lambda_{2}\right|$. Then $\varepsilon=\lambda_{0}-\lambda_{3}<\left|\lambda_{1}-\lambda_{2}\right| \leq \lambda_{1}+\lambda_{2}=1-\lambda_{0}-\lambda_{3}=-2 \delta+\varepsilon$. Hence $-2 \delta>0$. This is a contradiction.

- $\left|\lambda_{0}-\lambda_{3}\right|=\left|\lambda_{1}-\lambda_{2}\right|$ implies $2 \max _{i} \lambda_{i}=1-2 \min _{i} \lambda_{i}$.

- $\lambda_{0}+\lambda_{3}=\lambda_{1}+\lambda_{2}$ implies $2 \max _{i} \lambda_{i}=1-2 \min _{i} \lambda_{i}$.

Thus $\max _{i} \lambda_{i} \geq \frac{1}{2}$ implies that $\tau(\mathfrak{A}(\rho))=2 \max _{i} \lambda_{i}$. Therefore we conclude that $\tau(\mathfrak{A}(\rho)) \leq 1$ if and only if $\rho$ is separable.

Conclusion of the proof of Theorem 12: The remaining inequality in the proof of Theorem 12 now follows immediately from $\tau(\mathfrak{A}(\rho)) \leq\|\rho\|_{\gamma}$ and the results of this subsection.

\section{F. A two qutrit example}

Consider $\mathbb{C}^{3} \otimes \mathbb{C}^{3}$ and let $\{|0\rangle,|1\rangle,|2\rangle\}$ be the canonical real basis in $\mathbb{C}^{3}$. Consider the following family of qutrit mixed states defined on $\mathbb{C}^{3} \otimes \mathbb{C}^{3}$

$$
\rho_{\alpha}:=\frac{2}{7}\left|\Psi_{(3)}^{+}\right\rangle\left\langle\Psi_{(3)}^{+}\right|+\frac{\alpha}{7} \sigma_{+}+\frac{5-\alpha}{7} \sigma_{-},
$$

where we restrict ourselves to the parameter range $2 \leq \alpha \leq 5$, and where

$$
\begin{aligned}
\left|\Psi_{(3)}^{+}\right\rangle & \equiv \frac{1}{\sqrt{3}}(|0\rangle|0\rangle+|1\rangle|1\rangle+|2\rangle|2\rangle), \\
\sigma_{+} & \equiv \frac{1}{3}(|0\rangle|1\rangle\langle 0|\langle 1|+| 1\rangle| 2\rangle\langle 1|\langle 2|+| 2\rangle| 0\rangle\langle 2|\langle 0|) \\
\sigma_{-} & \equiv \frac{1}{3}(|1\rangle|0\rangle\langle 1|\langle 0|+| 2\rangle| 1\rangle\langle 2|\langle 1|+| 0\rangle| 2\rangle\langle 0|\langle 2|) .
\end{aligned}
$$

It is known [24] that $\rho_{\alpha}$ is (i) separable if and only if $2 \leq \alpha \leq 3$, (ii) bound entangled if and only if $3<\alpha \leq 4$ and (iii) entangled and distillable if and only if $4<\alpha \leq 5$.

We have 


$$
\begin{aligned}
\mathfrak{A}\left(\rho_{\alpha}\right)= & \frac{2}{21}\left(\left|E_{00}\right\rangle\left\langle E_{00}|+| E_{01}\right\rangle\left\langle E_{01}|+| E_{02}\right\rangle\left\langle E_{02}|+| E_{10}\right\rangle\left\langle E_{10}|+| E_{11}\right\rangle\left\langle E_{11}\right|\right. \\
& \left.+\left|E_{12}\right\rangle\left\langle E_{12}|+| E_{20}\right\rangle\left\langle E_{20}|+| E_{21}\right\rangle\left\langle E_{21}|+| E_{22}\right\rangle\left\langle E_{22}\right|\right) \\
& +\frac{\alpha}{21}\left(\left|E_{00}\right\rangle\left\langle E_{11}|+| E_{11}\right\rangle\left\langle E_{22}|+| E_{22}\right\rangle\left\langle E_{00}\right|\right) \\
& +\frac{5-\alpha}{21}\left(\left|E_{11}\right\rangle\left\langle E_{00}|+| E_{22}\right\rangle\left\langle E_{11}|+| E_{00}\right\rangle\left\langle E_{22}\right|\right) .
\end{aligned}
$$

Accordingly

$$
\begin{aligned}
\mathfrak{A}\left(\rho_{\alpha}\right)^{\dagger} \mathfrak{A}\left(\rho_{\alpha}\right)= & \frac{4}{441}\left(\left|E_{01}\right\rangle\left\langle E_{01}|+| E_{02}\right\rangle\left\langle E_{02}|+| E_{10}\right\rangle\left\langle E_{10}|+| E_{20}\right\rangle\left\langle E_{20}|+| E_{12}\right\rangle\left\langle E_{12}|+| E_{21}\right\rangle\left\langle E_{21}\right|\right) \\
& +\frac{2 \alpha^{2}-10 \alpha+29}{441}\left(\left|E_{00}\right\rangle\left\langle E_{00}|+| E_{11}\right\rangle\left\langle E_{11}|+| E_{22}\right\rangle\left\langle E_{22}\right|\right) \\
& +\frac{10+5 \alpha-\alpha^{2}}{441}\left(\left|E_{00}\right\rangle\left\langle E_{11}|+| E_{00}\right\rangle\left\langle E_{22}|+| E_{11}\right\rangle\left\langle E_{00}|+| E_{11}\right\rangle\left\langle E_{22}\right|\right. \\
& \left.+\left|E_{22}\right\rangle\left\langle E_{00}|+| E_{22}\right\rangle\left\langle E_{11}\right|\right) .
\end{aligned}
$$

The corresponding Eigenvalue problem can readily be solved using Ferrers' formula and we arrive at

$$
\tau\left(\mathfrak{A}\left(\rho_{\alpha}\right)\right)=\frac{19}{21}+\frac{2}{21} \sqrt{19-15 \alpha+3 \alpha^{2}} .
$$

It is easy to see that $\tau\left(\mathfrak{A}\left(\rho_{\alpha}\right)\right) \leq 1$ if and only if $2 \leq \alpha \leq 3$, i.e., if and only if $\rho_{\alpha}$ is separable. This example shows that there are inseparable (bound entangled) states which violate Equation (21) but satisfy the reduction criterion.

\section{G. Concluding remarks}

It is known that for $d \geq 3$ all inseparable Werner states violate the Peres-Horodecki positive partial transpose (ppt) criterion for separability (see [5,6]) but do not violate the reduction criterion introduced in [7]. As moreover the bound entangled states in subsection IIIH satisfy the ppt criterion, it follows from our results in subsections $\overline{\mathrm{III}}$ and $\mathrm{IIIF}$ that the separability criterion in Equation (21) is neither stronger nor weaker than the positive partial transpose criterion. Moreover, it also follows that the criterion Equation (21) is not weaker than the reduction criterion for separability. By the results of [9] this also implies that our criterion is not weaker than the entropic separability criteria based on the generalized Rényi and Tsallis entropies. The example in subsection III D implies that the separability criterion in Equation (21) is also not weaker than the criterion proposed by Nielsen and Kempe in [8] (as the criterion in [8] completely characterizes the separability properties of isotropic states in arbitrary dimension, but fails for the states $\rho_{p}$ discussed in subsection IIID and for all inseparable Werner states in dimension $d \geq 3$, see also [9]). Finally, violating our criterion does not imply distillability. 
Acknowledgement Thanks to the members of the Quantum Optics \& Information Group at Pavia for their hospitality and in particular to Giacomo Mauro D'Ariano and Shashank Virmani for helpful discussions about entanglement and about quantum information in general. Funding by the European Union project ATESIT (contract IST-2000-29681) is gratefully acknowledged.

[1] Rudolph O 2000 A separability criterion for density operators J. Phys. A: Math. Gen. 33 3951-3955

[2] Rudolph O 2001 A new class of entanglement measures J. Math. Phys. 42 2507-2512

[3] Vollbrecht K G H and Werner R F 2001 Entanglement measures under symmetry Phys. Rev. A 64062307

[4] Terhal B M and Vollbrecht K G H 2000 The Entanglement of Formation for Isotropic States Phys. Rev. Lett. 85 2625-2628

[5] Peres A 1996 Separability criterion for density matrices Phys. Rev. Lett. 77 1413-1415

[6] Horodecki M, Horodecki P and Horodecki R 1996 Separability of mixed states: necessary and sufficient conditions Phys. Lett. A 78 1-8

[7] Horodecki M and Horodecki P 1999 Reduction criterion of separability and limits for a class of protocols of entanglement distillation Phys. Rev. A 59 4206-4216

[8] Nielsen M A and Kempe J 2001 Separable states are more disordered globally than locally Phys. Rev. Lett. 86 5184-5187

[9] Vollbrecht K G H and Wolf M M 2002 Conditional entropies and their relation to entanglement criteria Preprint quant-ph/0202058

[10] Vidal G and Tarrach R 1999 Robustness of entanglement Phys. Rev. A 59 141-155

[11] Schmidt E 1907 Zur Theorie der linearen und nichtlinearen Integralgleichungen. I. Teil: Entwicklung willkürlicher Funktionen nach Systemen vorgeschriebener Math. Ann. 63, 433-476

[12] Ekert A and Knight P L 1995 Entangled quantum systems and the Schmidt decomposition Am. J. Phys. 63, 415-423

[13] Schatten R 1970 Norm Ideals of Completely Continuous Operators 2nd edn. (Berlin: Springer)

[14] Wegge-Olsen NE 1993 K-Theory and $C^{*}$-algebras (Oxford: Oxford University Press)

[15] Kadison R V and Ringrose J R 1983 \& 1986 Fundamentals of the Theory of Operator Algebras I \& II (Orlando: Academic Press)

[16] Werner R F 1989 Quantum States with Einstein-Podolsky-Rosen correlations admitting a hiddenvariable model Phys. Rev. A 40 4277-4281

[17] Horodecki R and Horodecki M 1996 Information-theoretic aspects of inseparability of mixed states Phys. Rev. A 54 1838-1843

[18] Bennett C H, DiVincenzo D P, Smolin J A and Wootters W K 1996 Mixed state entanglement and quantum error correction Phys. Rev. A 54 3824-3851

[19] Vidal G and Werner R F 2001 A computable measure of entanglement Preprint quant-ph/0102117.

[20] D'Ariano G M, Lo Presti P and Sacchi M F 2000 Bell measurements and observables Phys. Lett. A 272 32-38

[21] D’Ariano G M and Lo Presti P 2001 Optimal nonuniversally covariant cloning Phys. Rev. A 64042308

[22] Ferrers N M 1855 Two elementary theorems in determinants Quarterly Journ. of Math. i Decembre 
1855, p. 364 or Nouv. Annales de Math. xvi 402-403; ibid. xvii 190-191; cited after [23].

[23] Muir T 1911 The Theory of Determinants in the Historical Order of Development Vol. Two, p. 140; (reprinted by Dover 1960).

[24] Horodecki P, Horodecki M and Horodecki R 1999 Bound entanglement can be activated Phys. Rev. Lett. 82 1056-1059 